\title{
Role of Adjuvants in Vaccine Preparation: A Review
}

\author{
Pushpa Makwana*, Irshadullahkhan Kalyani, Dhruv Desai, Dharmesh Patel, \\ Pramod Sakhare and Dushyant Muglikar
}
Department of Veterinary Microbiology, College of Veterinary Science and Animal Husbandry, Navsari Agricultural University, Navsari-396445, Gujarat, India

*Corresponding author

\begin{abstract}
A B S T R A C T
\section{Keywords}

Adjuvants, Immunogen, Vaccine, Next generation adjuvants, Synthetic adjuvants

Article Info

Accepted:

10 October 2018

Available Online:

10 November 2018

Adjuvants are most important component of vaccine usually added with the immunogen in vaccine preparation. Since long back, adjuvants have been used to reduce immunogen dose, improve longevity and enhance the efficacy of vaccine. Though its wide use in pharma industries for vaccine manufacturing, it produce side effects, sometimes potent adverse reaction. From the earlier foundation of adjuvant activity, still there are limited resources of adjuvant have been frequently used by manufacturer. Role of adjuvants are most important factor for a particular vaccine preparation. With best possible way, adjuvants can act as key role in vaccines. Biological products, synthetic available substances and ethno-medicinal and pharma sources need to be explore for finding out newer adjuvants. Most of the next generation adjuvants are in clinical trials. Here, we have reviewed all possible, currently available and commonly used adjuvants with newly developed and recently tested adjuvant. Hence, we have put some light on emphasis of adjuvant research and development, as there is need of potent, safe, efficacious and nonreactogenic substances that can be used as adjuvant.
\end{abstract}

\section{Introduction}

The word "adjuvant" is actually derived from the Latin word adjuvare, meaning, "to help" or "to aid". Adjuvants are agents which added to vaccine formulation procedure that enhance the immunogenicity of antigen, help in activation of immune system and give protection against infection by long term protective immunity.(Lee and Minh, 2015). Adjuvants are immunomodulators of immune system and contains a diverse range of substances. Gaston Ramon, back in 1925 where he showed that co-administration of his newly invented diphtheria toxoid together with other compounds such as tapioca, lecithin, agar, starch oil, saponin or breadcrumbs increased antitoxin responses to diphtheria (Christensen, 2016).

Several existing vaccines consist of live attenuated pathogen, completely inactivated organism, recombinant protein as well as polysaccharide antigen. Due to its design, traditional vaccines possess inherent adjuvant activity (inherent immuno-stimulatory microbial structure as Pathogen Associated Microbial Pattern-PAMPs) which correlates 
with high immunogenicity (Liang and Lore, 2016). Although attenuated and killed vaccines are responsible for certain adverse reactions. To avoid nonspecific immunogenicity and toxicity, modern vaccine development has turned towards the application of highly purified, subunit, and synthetic antigens as vaccines. These vaccines are typically poor in immunogenicity due to specific antigen selection so, to enhance their immunogenicity certain additional components are required. These components are termed as adjuvants (Lombard et al., 2007).

\section{History}

Gaston Ramon observed that the yield of tetanus and diphtheria antibodies from horses were higher that had abscess formation at the injection site by injecting various components like starch, breadcrumbs or tapioca (Christensen, 2016). He induced sterile abscesses at injection site with inactivated toxin, and thus able to enhance anti-sera production, confirming the hypothesis that substances that have ability to produce local inflammation at the injection site were also able to enhance anti-sera production (Pasquale et al., 2015). Around the same time in 1926, Alexander Glenny and his colleagues observed the adjuvant activity of aluminum compounds using suspension of alum precipitated diphtheria toxoid (Lee and Minh, 2015).

In 1937, Jules Freund prepared water in oil (w/o) type emulsion adjuvant using paraffin oil mixed with killed mycobacteria called Freund Complete Adjuvant (FCA) and without mycobacteria referred as Freund Incomplete Adjuvant (FIA). Previously, FIA was used in human vaccines such as influenza and killed poliomyelitis vaccines. (Davenport, 1968;Salk and Salk, 1977). However, FIA is not currently in use because of its poor immunogenic effect, local irritation effect, granuloma and cyst formation at the site of injection and has carcinogenic activity in mice (Murray et al., 1972; Potter and Boyce, 1962; Gupta et al., 1993). The FCA strongly induce Th1 and Th2 immune response because of the combination of Mycobacterium tuberculosis with oil emulsion (Freund et al., 1948) but it inducing toxic effect that is major drawback of these adjuvants.

\section{Role of adjuvants}

Generally adjuvant is added to antigen to enhance the immunogenicity, additionally it helps to achieve a range of more specific effect. Addition of adjuvant in vaccine increases the antigen response, antibody titer (Podda, 2001; Beran, 2008) seroconversion rates and facilitate the use of smaller doses of antigen (Boyle et al., 2007; Banzhoff et al., 2009; Schwarz et al., 2009) which could be the important advantage in large-scale vaccine production.

To achieve desired protection many vaccines required multiple injections, which causes compliance issues. It can be reduced by adjuvant and reduce the number of doses required to achieve protection (Halperin et al., 2006; Banzhoff et al., 2009; Schwarz et al., 2009). It also increases speed of initial response, which may be critical in a pandemic outbreak of infection (Huleatt et al., 2007; Galli et al., 2009).

\section{Classification of adjuvants}

Adjuvants are classified according to its nature of origin, particle size and mechanism of action.

\section{Classification based on nature of origin}

Mineral compounds (e.g. Aluminum phosphate, Aluminum hydroxide and Alum), 
Bacterial compounds (e.g. Mycobacterial compounds, Muramyl dipeptide (MDP) and Lipopoly saccharide-LPS),

Oil based compounds like FCA, FIA, Starch oil and Saponin,

Immuno Stimulating Complexes (ISCOMs) (Cruz-Bustos et al., 2012; Aiyer et al., 2013).

\section{Classification based on their particle size}

Particulate (e.g. Aluminum salts, ISCOMs, w/o emulsions, Liposomes, Nanoparticles and micro particles).

Non-particulate antigen (e.g. MDP, Saponin, Lipid A, Bacterial toxins, polysaccharide and cytokines (Gupta and Chaphalkar, 2015).

Based on their principal mechanisms of action they are broadly classified

Antigen delivery systems (e.g. Alum, Liposomes, Virosomes, MF 59, Microparticles and nanoparticles),

Immunomodulators/ Immunostimulants (e.g. Monophosphoryl lipid A (MPL), CpG oligodeoxynucleotides (CpG ODN), Flagellin, Saponin, ds RNA and resiquimod).

\section{Mechanism of action}

Different Adjuvants have different mechanism of action. As the advancement in vaccine development, it is necessary to explore different adjuvant and their mechanism of action. Following are the varying mechanisms (Awate et al.,2013).

Adjuvants, like alum and emulsion (e.g. MF5 $\left.{ }^{\circledR}\right)$, It generates depots formation that trap antigen at the site of inoculation, slow down release of antigen and therefore provides continuous stimulation to the immune system. These adjuvants sustain the antigen at the injection site and enhance recruitment of immune cells.

Other adjuvants like ligands for pattern recognition receptors (PRR), either cell surface receptors or endosomal receptors, they act by inducing the innate immunity by targeting the APCs and influencing the adaptive immune response. Members belongs to PRR families like TLR, NOD like receptors (NLR), RIG-I-like receptors and Ctype lectin receptors (CLRs) all are potential targets for adjuvants. It exerts its effect by signaling through pathways that involve distinct adaptor molecules leading to the activation of different transcription factors. These transcription factors like, nuclear factorkappa beta (NF-kB), interferon regulatory transcription factor induce the production of cytokines and chemokines that play a key role in the priming, expansion and polarization of the immune responses.

Activation of some members of the NLR family triggers the formation of a protein complex, called inflammasomes, leads to caspases activation. Caspases are a family of protease enzymes that plays important roles in apoptosis and inflammation. It activates pro-inflammatory cytokines IL-1 $\beta$ and IL-18 that act as signals to immune cells and make the environment favorable for recruitment of immune cells to the site of damage and thus, has a fundamental role in the innate immune system.

Adjuvants like MF59 ${ }^{\circledR}$ induced recruitment of neutrophils, monocytes, eosinophils, macrophages followed by dendritic cells. The recruited cells especially neutrophils, monocytes, and B cells take up both antigen and adjuvant and traffic to draining lymph nodes to interact with antigen specific $B$ or $T$ cells to activate potent antibody secreting $\mathrm{B}$ cells and effector CD8+ T cells. 
Some adjuvants may exert their effect by antigen processing and presentation to Major Histocompatibility Complex (MHC). By this way, antigens activate $B$ cells by activation of $\mathrm{T}$ helper (Th) cells, primarily $\mathrm{Th} 1$ and Th2 cells. Th1 cells secrete IFN- $\gamma$, which activates macrophages and produce antibodies by $\mathrm{B}$ cells. The Th1 activates cytotoxic $\mathrm{T}$ lymphocytes which gives protection against intracellular pathogens (intracellular bacteria, protozoa and viruses) and induce death of cells. Natural killer cells play important role in the tumor apoptosis and virus infected cells and these (NK) cells are activated by the Th1 response.Th2 cells secrete cytokines, IL-4, which causes proliferation of $\mathrm{B}$ cells to make neutralizing antibodies. These Th2 cells augment a humoral (antibody) response against extracellular pathogens (helminthes, extracellular microbes and toxins) (Reed et al., 2013).

\section{Characteristics and adverse side effects of adjuvants}

Safety issue related with adjuvant is the important criteria for routine vaccination, Edelman (1980) listed various criteria like general toxicity, allergic reactions, carcinogenicity, teratogenicity, etc. Therefore, the ideal adjuvant should not induce toxicity, biodegradable, inexpensive and non reactogenic by itself and must not cause any reaction with the antigen (Sivakumar et al., 2011) Sometimes adjuvants can cause the adverse effects due to the interaction of the specific adjuvant and antigen. Adjuvants cause local reaction including pain, inflammation, ulceration and more rarely granulomas or sterile abscesses. A systemic nonspecific adverse effect includes fever, arthritis, uveitis, anorexia, soreness, and lethargy. Certain abnormal disease conditions are also associated with the use of adjuvants like of autoimmune disease condition, collagen disease and cancer (Dirga Sakti et al., 2015).

\section{Necessity for new adjuvants}

Since the discovery of adjuvant, aluminum salt work excellently for traditional bacterial toxoid and many of the currently available vaccines like diphtheria, tetanus, poliomyelitis, small pox and measles for which antibodies are the main key factor for protection. Though both alum and oil emulsion are poor inducer of cell-mediated immunity, they are not effective against infection caused by intracellular pathogen.

Second, there is a clear need for the development of vaccines against a number of infectious diseases such as Human immunodeficiency virus (HIV), hepatitis C virus (HCV), Respiratory Syncytial Virus (RSV), Neisseria meningitides serotype B, Group A \& B Streptococcus, Tuberculosis (TB) and malaria. New vaccines are also needed for protection against a number of emerging or re-emerging infectious diseases including Severe acute respiratory syndrome (SARS), Ebola, Hanta, Nipha, Zika and Dengue viruses (Derek,2007). Traditional vaccines do not protect against such pathogens, hence novel effective vaccines are required but these vaccines are poorly immunogenic. So to enhance new generation vaccines efficacy is to add highly purified synthetic adjuvants, which will activate specific immune response without showing side effects. Hence, the developments of new generation adjuvants are required.

Recombinant proteins as well as newer vaccines like subunit and DNA vaccines have very low immunogenicity. As there is advancement in vaccine development, also there is a necessity of development of newer adjuvant. (Sérgio Jorge and Odir Antônio Dellagostin, 2017) also there is a need of adjuvant that provides the agonistic effect with the newer vaccines. 


\section{Newer adjuvants}

In interest to reduce vaccine related adverse effects and to induce necessary adaptive immunity, numerous novel adjuvants are tested. Such as, liposomes and related structures, Saponin and Immune-Stimulating Complexes (ISCOMs), Nanoparticles and Micro particles, Bacterial derived adjuvants, CpG DNA, Virus Like Particles, Viral vectored vaccines and Toll Like Receptors (TLRs) (Brito and O'Hagan, 2014)

\section{Liposomes}

Liposomes are synthetic spheres containing lipid layers that can encapsulate antigens that are desired and act as adjuvants (Sivakumar, et al., 2011). Classical liposomes are vesicles composed of phospholipids and often cholesterol. They act by slowly releasing encapsulated antigen on intramuscular injection and by passively accumulating within regional lymph nodes. Liposomes can induce both humoral and cellular immunity to protein and polysaccharide antigens. The potency of liposomes varies with the number of lipid layers, electric charge, composition and method of preparation. The liposomal based vaccines fuse with cell membranes of macrophages, enabling the delivery of proteins into the cytoplasm and enter in to major histocompatibility complex (MHC) class I pathway and activate CD8 cells. The major drawback of liposomes is manufacturing difficulties such as stability, high cost and may produce pain at the site of injection. Recently, liposome based vaccines against Lung cancer(2011) is in phase III while vaccines against Neisseria meningitidis (2012), HIV(2013) and TB (2014) is in phase I clinical trial (Tandrup et al., 2016).

\section{Archaeosomes}

It is liposomes made with lipids from the
Archaea, act as good adjuvants. The Archaea belongs to a separate domain that is distinct from both bacteria and eukaryotes (Krishnan et al., 2000). Archaea often thrive in extreme environmental conditions and contain atypical lipids that can prepare stable liposomes. This stability may give good memory responses to included antigens. Lipids from Archaea vary in their efficiency, some can induce much higher titer than alum and some lipids also seem to be immunomodulators. Archaeosomes can enhance both Th1 cytokines (INF-gamma) and Th2 cytokines (IL-4), as well as cell-mediated responses to several antigens. The safety concerns of archaeosomes is still in evaluation process still there is no adverse effects have been seen. This lack of adverse effects are due to some Archaea can be found in mammalian hosts. Methanobrevibacter smithii, is a normal resident in the GI tract of humans is used to make archaeosomes. (Spickler and Roth, 2013).

\section{Saponin}

Saponin is the complex chemical adjuvant extracted from the tree Quillaia saponaria. The crude extract of this tree is called saponin (Glauert et al., 1962). Quil A is partially purified mixture, and Quillaia saponin fraction 21 (QS21) is defined fraction. Quil A is widely used in veterinary medicine and has been used in vaccines for cattle, pigs, horses, dogs, and cats including equine influenza virus, canine parvovirus, and Feline leukemia virus (FeLV) vaccines. QS21 is used in a FeLV vaccine and a canine Lyme disease vaccine. Saponins are immunomodulators and can induce strong Th1 and Th2 responses as well as CTL. Generally, it is safe, but its relative safety may depend on the route of administration, the species, and the specific Saponin. Intra-venous injection of less purified fraction can result in toxicity, probably due to hemolysis. Injection of free 
Quil A is well tolerated in sheep and cattle, but some toxicity has been reported in cat. Local inflammatory reactions occur with free Quil A but can be suppressed without loss of adjuvant activity when it is combined with cholesterol-containing liposomes. Purified Saponin fractions have a much lower toxicity than Quil A and are being considered for vaccine in humans. Currently QS-21 evaluated against cancer, HIV and malarial antigens, which is in phase I and II clinical trials (Gupta andChaphalkar, 2015).

\section{Immune-Stimulating Complexes (ISCOMs)}

These novel particles or complexes are known as ISCOMs. Itis first describe by Morein and coworkers in 1984. The classic ISCOMs formed by the combination of cholesterol, Saponin, phospholipid, and amphipathic proteins (Morein et al., 1984; Cruz-Bustos et al., 2012). The second is ISCOM Matrix. Typically, both ISCOMs and Matrix exist as spherical, hollow, rigid, cage-like particles of about $40 \mathrm{~nm}$ in diameter with a strong negative charge. The basis of the unique structure of ISCOMs is the interaction between Saponin and cholesterol molecules, which combined and form stable ring. The individual cholesterol-saponin rings combine to form pentagonal structure (much like a soccer ball) and are held together by hydrophobic interactions, steric factors, and possibly hydrogen bonds. Phospholipid (typically egg-derived phosphatidylcholine) is needed when protein is to be incorporated into an ISCOM structure. It has been suggested that this promotes a less rigid structure than saponin and cholesterol alone and thus allows the bulky amphipathic molecules (e.g., viral membrane proteins) to insert into the ISCOM structure. In veterinary vaccines, the saponin sometimes is Quil A, but more purified fraction is used in vaccines for humans. ISCOMs are immunomodulators. It can induce Th1 reactions and CTLs as well as concurrent
Th2 responses in some circumstances. It have been used with more than 20 viral, bacterial, and parasitic pathogens and are found in experimental bovine viral diarrhea virus, bovine herpes virus type 1, rinderpest, FeLV, pseudorabies, and canine distemper vaccines. An equine influenza vaccine with an ISCOM adjuvant currently is marketed for horses in Europe. ISCOMs are also being tested for use in vaccines for humans. It Promote antibody responses and induce $\mathrm{T}$ helper cells as well as CTL responses in experimental animal models, now in phase I and II human trials.

A further attraction of ISCOMs is its reduced toxicity and reactogenicity compared with other saponin-based formulations. ISCOMs have been made with amphipathic molecules derived from many sources including cell wall and membrane proteins from viruses such as Herpes simplex virus -1(HSV-1), Cytomegalovirus (CMV), hepatitis $\mathrm{B}$, rabies and influenza. An influenza ISCOM vaccine for horses is licensed in Europe. When administered intranasally, influenza ISCOMs vaccines were found to elicit strong mucosal (IgG and $\operatorname{IgA}$ ) responses. Recently vaccines in clinical trials for influenza, melanoma and HCV (Lee and Nguyen 2015).

\section{Nanoparticles and microparticles}

Nanoparticles and microparticles are made from biodegradable polymers, like cyanoacrylates and poly (lactide-co-glycolide) copolymers. Nanoparticles $(10-1,000 \mathrm{~nm})$ are of nanometer in size while microparticles (1$100 \mathrm{~mm}$ ) are of micrometer in their size. These nontoxic polymers that are used in adjuvants are used as suture material, prostheses, and drug carriers. Studies of these adjuvants reveals no serious adverse effects. One of the important characteristics of these adjuvants is formation of a long-term depot that favours release of antigen for longer periods upto several months. Microparticles 
that have both characteristics fast-releasing and slow-releasing could produce both primary and booster immunization with single injection (Anna and James, 2003).

Antigens that are incorporated along with microparticle protected from adverse conditions like low $\mathrm{pH}$, bile salts, and enzyme activities. Due to this property, it may be particularly useful in oral and intranasal vaccines. During manufacturing, encapsulation process may modify antigen and decrease its ability to stimulate the immune system that limits the use of this adjuvant. However, novel techniques and types of particles may overcome this issue. Nanoparticles and microparticles are being tested in companion animals, in horse, as well as in cattle, swine, and fish (Spickler A. and Roth, 2003).

\section{Bacterially derived adjuvants}

\section{Toxins}

\section{Cholera toxin}

Cholera toxin (CT) is a protein complex secreted by the bacterium Vibrio cholerae. Cholera toxin is responsible for the massive, watery diarrhoea characteristic of cholera infection. Cholera toxin has been proven to enhance the immunogenicity of relatively poor mucosal immunogen, when mixed or conjugated together and give intranasally, it generate great potent immune response.

Thus, it used as a potential adjuvant for oral vaccine. Even adjuvant potential of CT $\beta$ subunit is widely tested with vaccine through intra-muscular injection in mice (Heidelberg et al., 2000).

\section{Pertussigen}

The killed Bordetella pertussis bacterium has been used experimentally as a parenteral adjuvant. Obviously, this material is a complex mixture including LPS as well as variable amount of pertussis toxin (PT). In particular, it enhances the cellular immune response as measured by delayed skin test responses to soluble antigens and increases inflammatory responses such as footpad swelling after injection. Like LPS, pertussigen can be given by a different route at a different time and still exerts its adjuvant effect (Roberts et al., 1995)

\section{Shiga toxin}

Shiga toxin (STx) is a protein toxin of Shigella dysenteriae, Type-I, a causative agent of severe diarrhoea (Tarr et al., 2005). Mice vaccinated oro-gastrically with various doses of STx developed serum and gastro-intestinal antibodies to STx in a dose dependent manner. In a recent study, the immunomodulatory potential of recombinant Shigatoxin B subunit $(\mathrm{rStxB})$ protein in $\mathrm{BALB} / \mathrm{c}$ mice was evaluated. Animal protection with recombinant StxB was conferred through both humoral and cellular immune response. Such vaccines are in clinical trials for influenza and melanoma (Lee and Nguyen 2015).

\section{Non-toxin proteins}

\section{Muramyl dipeptide (MDP)}

$\mathrm{N}$-aceylyl muramyl-L-alanyl-D-isoglutamine is derived from the cell wall of mycobacteria (Bolhassani et al., 2017) it is the smallest structural component of the cell wall that still retains adjuvant activity and is one of the active components in FCA. In several instances, oral administration of MDP has been used to stimulate non-specific immunity to bacteria and tumour cells. The increase in non-specific immunity was probably due to induction of cytokine. MDP is known to be a potent inducer of interleukin-1 (IL-1), which can activate macrophages and T-cells. 
Although, not directly relevant to mucosal immunity but it show that MDP is absorbed by the gut and, therefore, may affect the immunoregulatory environment of mucosaassociated lymphoid tissue (MALT). The mechanism of action is unknown, but is probably due to the ability of MDP to induce basophils production and increase processing and presentation of antigens by macrophages (Mohan et al., 2013).

\section{CpG Deoxynucleotides}

Bacterial DNA can act as an adjuvant and induce cytokine release. $\mathrm{CpG}$ oligonucleotides (ODNs) are adjuvants that mimic the bacterial DNA motif that is represent in vertebrate DNA. These oligonucleotides contain a central unmethylated $\mathrm{CpG}$ dinucleotide in the context of particular base sequence ( $\mathrm{CpG}$ motifs) exert a strong stimulatory influence on the immune system. Such sequences either are found naturally in bacterial DNA or produce as synthetic oligonucleotides directly. B-cells and plasmacytoid dendritic cells activated via TLR9.

CpG oligoes act as polyclonal activator, directly activate B-cells to proliferate and differentiate into IgG producing cells. CpG oligoes also indirectly activate other cells such as monocytes and macrophages to produce a variety of pro-inflammatory cytokines and in particular, that associated with stimulatory influences. $\mathrm{CpG}$ were capable of enhancing $\mathrm{CD} 4^{+} \mathrm{T}$-cells, $\quad \mathrm{CD} 8^{+} \mathrm{T}$-cytotoxic cells and antibody response to a wide variety of antigens. Because of their strong adjuvant potency and low reactogenicity, CpG ODNs are currently considered as one of the most promising adjuvants for the development of future vaccines against diverse condition including infectious disease, allergies or cancer. Although low doses appear to be safe in some studies, repeated high doses of $\mathrm{CpG}$ ODNs can induce splenomegaly in mice, and bacterial DNA can cause cytokine release and fatal shock (Bode et al., 2011; Spickler and Roth, 2013).

\section{Virus like particles (VLPs)}

VLPs are essentially non-infective virus consisting of self-assembled vial envelope proteins without accompanying the genetic material (Gilbert, 2001). Virus like particles maintains a morphology and cell-penetrating ability similar to infective viral particles. The VLPs have also been shown to stimulate both cellular and humoral immunity. Several recombinant HBV-VLP (Hepatitis B virusVLP) vaccines have been licensed. The first licensed recombinant HBV vaccines; Recombivan $^{\mathrm{TM}}$ and energin- $\mathrm{B}^{\mathrm{TM}}$, were composed of the viral small envelope protein which upon expression in yeast formed $22 \mathrm{~nm}$ VLPs. While effective, these suffered from a lack of immunogenicity (5-10\% nonresponders), which was determined to be due to an absence of pre-S epitopes on the surface of VLPs.

A more immunogenic VLP vaccine was subsequently described that contained Pre-S1, Pre-S2 and HBV surface antigen. This potential third generation HBV vaccine, Bio$\mathrm{HepB}^{\mathrm{TM}}$ was found to elicit a strong antibody response and 100 per cent sero-conservation and sero-protection rates.

One recently approved VLP vaccine is Gardozil $^{\mathrm{TM}}$ for immunization against human papilloma virus (HPV) and subsequent prevention of cervical cancer. This vaccine is composed primarily of self-assembled particles of L1 (the major capsid protein) from HPV types 6, 11 and 18 and contain an aluminum salt adjuvant.

Table.1 TLRs commercial ligand and their Sources 


\begin{tabular}{|c|c|c|c|c|}
\hline TLRs & $\begin{array}{l}\text { LIGAND/ } \\
\text { COMMERCIAL } \\
\text { AGONIST }\end{array}$ & $\begin{array}{l}\text { COMERCIAL } \\
\text { SOURCE }\end{array}$ & ANTAGONIST & $\begin{array}{l}\text { COMMERCIAL } \\
\text { SOURCE }\end{array}$ \\
\hline TLR 2 & $\begin{array}{c}\text { Lipoglycans, } \\
\text { Lipoteichoic Acids, } \\
\text { Peptidoglcans, Synthetic } \\
\text { Lipoproteins, Zymosan }\end{array}$ & InvivoGen & $\begin{array}{l}\text { OxPAPC (TLR } 2 \text { and } \\
\text { TLR } 4 \text { inhibitor) }\end{array}$ & InvivoGen \\
\hline TLR 3 & $\begin{array}{l}\text { Virus ds RNA } \\
\text { /polyinosinic- } \\
\text { polycytidylic acid (Poly } \\
\text { I:C) }\end{array}$ & $\begin{array}{l}\text { Sigma- Aldrich, } \\
\text { InvivoGen }\end{array}$ & $\begin{array}{l}\text { 614310-TLR3/dsRNA } \\
\text { Complex Inhibitor - } \\
\text { Calbiochem }\end{array}$ & MerckMillipore \\
\hline TLR 4 & $\begin{array}{c}\text { Lipopolysaccharides, } \\
\text { Monophosphoryl Lipid } \\
\text { A, }\end{array}$ & $\begin{array}{l}\text { InvivoGen, } \\
\text { Sigma- Aldrich }\end{array}$ & $\begin{array}{l}\text { Lipopolysaccharide } \\
\text { from the } \\
\text { photosynthetic } \\
\text { bacterium } \\
\text { Rhodobacter } \\
\text { sphaeroides (LPS-RS) }\end{array}$ & InvivoGen \\
\hline TLR 5 & Flagellin & $\begin{array}{c}\text { InvivoGen, } \\
\text { Sigma- Aldrich }\end{array}$ & $\begin{array}{l}\text { Soluble ectodomain of } \\
\text { TLR5 of human with } \\
\text { engineered Fc region } \\
\text { fusion (hTLR5-Fc) }\end{array}$ & InvivoGen \\
\hline TLR 7/8 & $\begin{array}{l}\text { ssRNA /Resiquimod, } \\
\text { Imiquimod } \\
\text { VaxxiGrade }^{\mathrm{TM}}\end{array}$ & InvivoGen & - & - \\
\hline TLR 9 & $\begin{array}{c}\text { Unmethylated CpG DNA } \\
\text { (intracellular sensor of } \\
\text { bacterial DNA) /Class A } \\
\text { CpG ODNs (ODN 1585, } \\
\text { ODN 2216, ODN 2336); } \\
\text { Class B CpG ODNs, } \\
\text { Class C CpG ODNs }\end{array}$ & InvivoGen & ODN 2088 & InvivoGen \\
\hline TLR 13 & $\begin{array}{c}\text { 23S ribosomal RNA, } \\
\text { ORN Sa19 }\end{array}$ & InvivoGen & - & - \\
\hline
\end{tabular}

Table.2 Detail of emulsion

\begin{tabular}{|l|l|l|l|} 
& $\begin{array}{l}\text { Freund Incomplete } \\
\text { Adjuvant }\end{array}$ & $\begin{array}{l}\text { Water in Oil } \\
\text { emulsion (W/O) }\end{array}$ & $\begin{array}{l}\text { Oil in Water emulsion } \\
(\mathrm{O} / \mathrm{W})\end{array}$ \\
\hline Oil/Water ration & $50 / 50$ & $70 / 30$ & $30 / 70$ or $25 / 75$ or $20 / 80$ \\
\hline Droplet test identity & W/O based & W/O based & O/W based \\
\hline Stability at $4^{\circ} \mathrm{C}$ & Few weeks & $>2$ years & $>2$ years \\
\hline Stability at $20^{\circ} \mathrm{C}$ & Few weeks & $>2$ years & $>2$ years \\
\hline Stability at $37^{\circ} \mathrm{C}$ & Few weeks & $>3$ months & $>2$ months \\
\hline
\end{tabular}

Table.3 Adjuvants in licensed vaccine 


\begin{tabular}{|c|c|c|c|c|}
\hline $\begin{array}{l}\text { Adjuvant name } \\
\text { (year licensed) }\end{array}$ & Class & $\begin{array}{l}\text { Clinical } \\
\text { vaccines }\end{array}$ & Manufacturer & Description \\
\hline Alum (1926) & Aluminum salt & $\begin{array}{l}\text { DPT } \\
\text { (diphtheria, } \\
\text { Pertussis, and } \\
\text { Tetanus), } \\
\text { HBV, HAV }\end{array}$ & Various & $\begin{array}{l}\text { Improves humoral } \\
\text { immune response and } \\
\text { antigen stability. } \\
\text { Antigens are adsorbed } \\
\text { to the surface. The } \\
\text { adjuvant in }>80 \% \text { of } \\
\text { vaccines licensed for } \\
\text { human use. Th2 type } \\
\text { immune responses. }\end{array}$ \\
\hline MF59 (1997) & $\mathrm{o} / \mathrm{v}$ emulsion & $\begin{array}{l}\text { Seasonal and } \\
\text { pandemic flu }\end{array}$ & Novartis & $\begin{array}{l}\text { Improves humoral and } \\
\text { cell-mediated } \\
\text { immunity. Used in } \\
\text { influenza vaccines and } \\
\text { Antigen delivery. }\end{array}$ \\
\hline $\begin{array}{l}\text { Virosomes } \\
(2000)\end{array}$ & Liposome & $\begin{array}{l}\text { Seasonal flu, } \\
\text { HAV }\end{array}$ & $\begin{array}{l}\text { Berna Biotech } \\
\text { (Crucell } \\
\text { Biotech } \\
\text { company) }\end{array}$ & $\begin{array}{l}\text { Improves humoral and } \\
\text { cell-mediated } \\
\text { immunity. A } \\
\text { virosomes is the } \\
\text { reconstituted } \\
\text { membrane of an } \\
\text { enveloped virus. The } \\
\text { vaccines for influenza } \\
\text { and for Hepatitis A are } \\
\text { approved products. }\end{array}$ \\
\hline AS04 (2005) & $\begin{array}{l}\text { Alum-adsorbed } \\
\text { TLR4 agonist }\end{array}$ & $\begin{array}{l}\text { HPV-16/18, } \\
\text { HBV }\end{array}$ & GSK & $\begin{array}{l}\text { Improves humoral and } \\
\text { cell-mediated } \\
\text { immunity. } \\
\text { Combination of } \\
\text { aluminum adjuvant } \\
\text { with monophosphoryl } \\
\text { lipid A (MPL) co- } \\
\text { adsorbed. Used for } \\
\text { HPV and HBV } \\
\text { vaccine. }\end{array}$ \\
\hline AS03 (2009) & $\begin{array}{l}\mathrm{o} / \mathrm{w} \quad \text { emulsion } \\
\& \alpha \text {-tocopherol }\end{array}$ & Pandemic flu & GSK & $\begin{array}{c}\text { Improves humoral and } \\
\text { cell-mediated } \\
\text { immunity. Used in } \\
\text { influenza vaccine } \\
\text { during } 2009 \text { H1N1 } \\
\text { pandemic. }\end{array}$ \\
\hline $\begin{array}{l}\text { AS01 } \\
\text { (July,2015) }\end{array}$ & $\begin{array}{l}\text { MPL,QS21, } \\
\text { liposome }\end{array}$ & RTS,S malaria & GSK & $\begin{array}{l}\text { Induction of Antigen } \\
\text { specific CD4+ T cells }\end{array}$ \\
\hline \multicolumn{5}{|c|}{$\begin{array}{l}\text { It has shown to reduce infection of HPV by cent effective against these types since two of } \\
90 \text { per cent and is apparently almost } 100 \text { per } \\
\text { the four antigens in the HPV vaccine (HPV }\end{array}$} \\
\hline
\end{tabular}


types 16 and 18) are implicated in 70 per cent of the cervical cancer.

This vaccine is expected to drastically reduce the occurrence of the life threatening disease in women (Mohan et al., 2013).

\section{Toll like Receptors (TLRs)}

TLRs are pattern recognition receptors, which play an important role in microbial sensing (Akira et al., 2006). The PAMPs binding the TLRs are the basis of many adjuvants. Cell surfaces TLR are TLR1, TLR 2, TLR 4, TLR 5, TLR 6 and TLR 11 recognizing extracellular antigens while TLRs Within the cells like TLR 3, TLR 7, TLR 8, TLR 9 and TLR 10 detecting intracellular invader. TLR 13 has been identified in mouse but absent in human.

Stimulation of TLRs by the corresponding PAMPs initiate the signaling cascades leading to the activation of transcription factors, such as $\mathrm{AP}-1, \mathrm{NF}-\kappa \mathrm{B}$ and interferon regulatory factors (IRFs). Signaling by TLRs result in a variety of cellular responses including the production of interferons (IFNs), proinflammatory cytokines and effector cytokines that direct the adaptive immune response.

All TLRs except TLR 3 use an adopter protein called MyD88 to activate three major transcription factors, NF- $\kappa \mathrm{B}$, MAP kinase and IRF3. NF- $\kappa \mathrm{B}$ and MAPK activate the genes for three major proteins IL-1, IL-6 and TNFalpha as well as the activating enzyme caspase-1. The TRIF complex turns on another transcription factor IRF3 that activates the gene for the antiviral cytokine, Interferon- beta. (Kawai and Akira, 2010)

\section{TLR ligands}

It includes synthetic compounds that induce the maturation and activation of professional
APC and the secretion of inflammatory cytokines and chemokines (Akira et al., 2006). The small molecule nucleoside analogues imiquimod and resiquimod are ligands for TLR-7 and TLR-7/8, respectively. Imiquimod used for leishmaniosis, and is licensed for treatment of HPV and basal cell carcinoma (BCC). The exact mechanism ofimiquimod action is unknown. But it is thought that its activity as a TLR-7 agonist mimic a microbial antigen inducing the expression of different cytokines such as IL-1, IL-6, IL-12, IFN- $\alpha$ and TNF- $\alpha$, which stimulate or enhance both the innate immune system and the cell-mediated immune response, enhances migration of Langerhans' cells from the dermis to regional lymph nodes.

\section{Adjuvants in licensed vaccines}

Only few adjuvants are used in licensed vaccines due to their lower adverse effects and better adjuvant activity for example alum, MF59, Virosomes, AS04, AS03 and AS01 (Liang and Lore 2016)

\section{Alum (1926)}

Aluminum hydroxide and other aluminum salts (Aluminum hydroxyl-phosphate sulphate, Aluminum potassium phosphate, and others), typically referred to as "Alum", are the most widely used adjuvants in human and animal vaccines. Alum elicits strong humoral immune responses primarily mediated by secreted antigen-specific antibodies. Alum is a component of licensed human vaccines such as Hepatitis A virus (HAV), HBV, HPV, diphtheria, tetanus, Haemophilus Influenza Type b (Hib) and meningococcal where neutralizing antibodies to bacterial and viral antigens are required for protection. In contrast, alum is a poor inducer of cell-mediated immune responses and is unsuitable for vaccines that require a strong 
cellular immune response. The mechanism by which alum act as adjuvant is the formation of nodules at injection site act as depot site for the antigens and it allows slow release of antigen thus providing both a priming and a boosting effect with the same inoculation. Limitation of alum they are poor inducer of cell mediated immunity so unsuitable for vaccines that require a strong cellular immune response. Vaccine containing alum, which cannot sterilized by filtration, frozen or lyophilized (Lee and Nguyen 2015).

\section{Emulsions}

It consists of two or more immiscible liquids such as water and oil. The minor component (the dispersed phase) is dispersed in the predominant component (the continuous phase). Emulsions are stabilized with amphiphilic molecules, referred to as emulsifiers or surfactants. Generally, there are water in oil (w/o) and oil in water (o/w) type of emulsion are available. w/o emulsion type of emulsion is more potent efficient and generally used in vaccine preparation. As shown in table 2, Stability index of vaccine has been studied in various temperature effect. Stability of vaccine and temperature has direct correlation which might be influence by adjuvant (Aucouturier, et al., 2001).

\section{MF59 (1997)}

It is an $\mathrm{o} / \mathrm{w}$ emulsion made of squalene droplets in a continuous aqueous phase, with a diameter of $167 \mathrm{~nm}$. MF59 has been approved for the $\mathrm{H} 5 \mathrm{~N} 1$ pandemic influenza vaccine (Fluad ${ }^{\mathrm{TM}}$ ) and for the H1N1 influenza vaccine (Focetria ${ }^{\circledR}$ and Celtura ${ }^{\circledR}$ ) in Europe. It recruits monocytes and macrophages into injection site by the induction of local chemokine secretion. MF59 can also augment antigen uptake by dendritic cells (DCs) and activate CD4 $\mathrm{T}$ cells. As a result, MF59 generates high antibody titer with balanced IgG1:IgG2a responses.

MF59 has been evaluated in conjunction with HSV, HIV, HBV and CMV vaccine trials. It enhances immune responses in the elderly population and can facilitate immune responses against specific drift variants of the seasonal influenza virus not included in the vaccine (Lee and Nguyen 2015).

\section{Virosomes (2000)}

It is a liposome-based structure that have viral proteins embedded into the membrane, for example the hemagglutinin (HA) and neuraminidase proteins of an influenza virus.

These elements help to target the Virosomes to the immune system cells, delivering the vaccine antigen. The suggested mechanism of action of influenza-based Virosomes involves direct interaction of Virosomes particles with APCs or, in some cases, with B cells, which in turn, activate T cells.

It presents antigen via both MHCI and II, able to induce both humoral immunity and CMI The influenza HA antigen targets the Virosomes to APCs which engulf it by endocytosis and present the antigens to $\mathrm{T}$ cells after proteolytic degradation. Currently there are two Virosome-adjuvanted vaccines, licensed in some European and non-European countries.

\section{Adjuvant system in licensed vaccines}

Adjuvant system is a combination of two or more adjuvants designed for enhance and desired immune response. (Brito and O’Hagan, 2014, Del Giudice et al., 2018)

AS04 (2005)

Adjuvant System (AS) 04 (AS04), which is based on a LPS derivative, MPL and 
aluminum salts. LPS, derived from Gramnegative bacteria, is a potent immunostimulant and a specific TLR4 agonist. MPL is obtained by mild hydrolysis and further purification of LPS derived from Salmonella minnesota. The product has similar immunostimulatory properties to LPS, but lacks the reactogenicity of native LPS. In AS04, MPL is adsorbed onto aluminum hydroxide or aluminum phosphate, depending on the vaccine with which it is used.

In AS04, MPL plays a crucial role in the activation of the innate immune system. Direct stimulation of TLR4 leads to the maturation of APCs, inducing the expression of cytokines that in turn enhance the adaptive immune response by stimulating the maturation of Th cells, in particular Th1. Therefore, recognition of MPL by TLR4 leads to enhanced humoral and cellular immune response. AS04 has to be administered at the same injection site as the antigen together or within 24 hour to exert its effect. During this period, AS04 transiently induces local innate cell activation and cytokine production, as demonstrated by the local increase in activated antigen-loaded DCs and monocytes, which migrate to the lymph node draining the injection site. Aluminum salt appears to modulate and prolong the cytokine responses to MPL at the injection site. Taken together, these results support a model where the addition of MPL to aluminum salt enhance the vaccine response by prompting increased activation of APCs and downstream enhanced stimulation of Th1 T-cell responses.

\section{AS03 (2009)}

AS03 is a combination of adjuvants, $\alpha$ tocopherol (vitamin E) and squalene in an $\mathrm{o} / \mathrm{w}$ emulsion with a droplet diameter of 150-155 $\mathrm{nm}$. It is used in pandemic influenza vaccines. Vitamin $\mathrm{E}$ is a lipid-soluble antioxidant with immune-enhancing properties, which is present in the human body in muscles, adipose tissues, the adrenal and pituitary glands, and pancreas. The most important function of vitamin $E$ is to maintain the integrity of cellular membranes by protecting their physical stability, and by inhibiting tissue damage caused by oxidation.

Both monocytes and macrophages respond to AS03 with a local production of a range of cytokines and chemokines. Macrophages are the most likely initiators of the cytokine response, whereas recruited monocytes elicit a second wave of chemokine secretion and further innate cell recruitment. An AS03adjuvanted pandemic influenza vaccine has been shown to allow for antigen sparing and less antigen is needed per vaccine dose. In addition, a high level of cross-reactive immunity to heterologous strains of H5N1 has beenalso observed (Satoshi et al., 2014).

\section{AS 01 (2015)}

It consists of liposomes, MPL and QS21. Recently, it is approved for malaria vaccine that is recombinant protein-based malaria vaccine (Mosquirix ${ }^{\mathrm{TM}}$ ), resulted in a successful but modest efficacy in infants in seven malaria-endemic countries. Infection is prevented by inducing humoral and cellular immunity, high antibody titers which block the parasite from infecting the liver (Liang and Lore 2016).

\section{Problems for the development of novel adjuvant}

Several barriers must overcome to meet the demand for new adjuvant. The following issues remain considerable problem for the development of new adjuvant:

Unacceptable side effects and toxicity remain barriers for many candidates, particularly for the development of vaccine for neonates. Currently, adjuvant does not receive U.S. 
Food and Drug Administration (FDA) approval as standalone product, but as part of a registered vaccine adjuvant-antigen combination.

Therefore, potential adjuvant-antigen combination have not been developed because of the huge cost and effort involved in gaining FDA approval for each adjuvant-antigen combination.

Most for-profit-organization are unwilling to risk the investment in new vaccine that involve untested antigen and adjuvant.

Most vaccine companies keep their adjuvant formulation proprietary unexposed until the adjuvant registered with a potential vaccine product. This limits the development of the adjuvant for other vaccine application.

The high cost of developing novel adjuvant formulation is the major constraint.

A major challenge in adjuvant development is how to achieve a potent adjuvant effect while avoiding reactogenicity or toxicity, need to undertake for further research.

The effects of various adjuvants on T-cell response need to be evaluated and future work should carry out. Still, more research requires on other adjuvant receptors, too. That may provide adjuvant with differential properties for clinical use.

Recent successful clinical studies conducted with new adjuvants suggest that novel adjuvants will be utilized for human vaccine formulation in a near future. The availability of these adjuvants in various combinations will facilitate the rational design of vaccine against infectious disease.

Interest in alternative to alum and oil emulsion has led to increase availability of new adjuvant over the last decade. For FDA approval, new adjuvant must convincingly demonstrate safety, tolerability and no reactogenicity. Adjuvant currently used in animals and humans enhance the humoral immunity, but many new adjuvants are in clinical or pre-clinical development which are being focused on enhancing specific type of $\mathrm{T}$-cell response and generating the multifaceted immune response that may be needed for challenging diseases such as malaria and HIV. Well-defined ligands for PRR have attracted most of the attention. To select an adjuvant for a vaccine, it must be understood, "the best adjuvant is not always the same for all antigens" and situations. Most vaccine reactions are not life threatening and resolve over time, even rare serious adverse events discourage better community acceptance of routine vaccination. Vaccine funding agencies should consider and initiate specific funding programs for adjuvant science and clinical development; even it remains a major roadblock in new vaccine development, too.

\section{Conflicts of Interest}

The authors declare no conflicts of interest.

\section{Acknowledgement}

Authors are thankful to Dean and Vice chancellor for providing necessary facilities and funds.

\section{References}

Aiyer H., Kumar H., Gupta P. and Shivkumar N. (2013). An overview of immunologic adjuvants- a review. Journal of Vaccine and Vaccination. 4,1-4.

Aucouturier, J., Dupuis, L., and Ganne, V. (2001). Adjuvants designed for veterinary and human vaccines. Vaccine, 19(17-19), 2666-2672.

Awate, S., Babiuk, L. A. B. and Mutwiri, G. (2013). Mechanisms of action of 
adjuvants. Frontiers in immunology, 4, 114.

Banzhoff A., Gasparini R., Laghi-Pasini, F., Staniscia T., Durando P., Montomoli E., Capecchi P.L.,Giovanni, P. and Sticchi, L. (2009). MF59-adjuvanted H5N1 vaccine induces immunologic memory and heterotypic antibody responses in non-elderly and elderly adults. PLoS one 4, e 4384.

Beran J. (2008). Safety and immunogenicity of a new hepatitis $B$ vaccine for the protection of patients with renal insufficiency including pre-haemodialysis and haemodialysis patients. Expert Opinion in Biological Therapy.8, 235247.

Bode, C., Zhao, G., Steinhagen, F., Kinjo, T., and Klinman, D. M. (2011). CpG DNA as a vaccine adjuvant. Expert review of vaccines, 10(4): 499-511.

Bolhassani, A., Talebi, S., and Anvar, A. (2017). Endogenous and exogenous natural adjuvants for vaccine development. Mini reviews in medicinal chemistry, 17(15): 1442-1456.

Boyle J., Eastman D., Millar C., Camuglia S., Cox J., Pearse M., Good J. and Drane D. (2007). The utility of ISCOMATRIX adjuvant for dose reduction of antigen for vaccines requiring antibody responses. Vaccine 25, 2541-2544.

Brito, L. A. and O'Hagan, D. T. (2014). Designing and building the next generation of improved vaccine adjuvants. Journal of Controlled Release, 190: 563-579.

Christensen, D. (2016). Vaccine adjuvants: Why and how. Human vaccines and immunotherapeutics. 12, 2709-2711

Coffman R., Sher A. and Seder A. (2010). Vaccine adjuvants: putting innate immunity to work. Immunity.33, 492502.

Cruz-Bustos, T., González-González, G., Morales-Sanfrutos, J., Megía-Fernández, A., Santoyo-González, F., and Osuna, A. (2012). Functionalization of immunostimulating complexes (ISCOMs) with lipid vinyl sulfones and their application in immunological techniques and therapy. International journal of nanomedicine, 7, 5941

Cruz-Bustos, T., González-González, G., Morales-Sanfrutos, J., Megía-Fernández, A., Santoyo-González, F. and Osuna, A. (2012). Functionalization of immunostimulating complexes (ISCOMs) with lipid vinyl sulfones and their application in immunological techniques and therapy. International journal of nanomedicine, 7, 5941.

Del Giudice, G., Rappuoli, R., and Didierlaurent, A. M. (2018, May). Correlates of adjuvanticity: A review on adjuvants in licensed vaccines. In Seminars in immunology. Academic Press.

Edelman R. (1980). Vaccine adjuvants. Rev Infect Dis.2, 370-83.

Frank Liang and Karin Loré (2016). Local innate immune responses in the vaccine adjuvant-injected muscle. Clinical and Translational Immunology. 5

Freund J, Thomson KJ, Hough HB, Sommer HE, and Pisani TM. (1948). Antibody formation and sensitization with the aid of adjuvants. Journal of Immunology. 60, 383-398.

Galli G., Hancock K., Hoschler K., DeVos J., Praus M., Bardelli M., Malzone C., Castellino F., Gentile C. and McNally T. (2009). Fast rise of broadly cross-reactive antibodies after boosting long-lived human memory B cells primed by an MF59 adjuvanted prepandemic vaccine. Proc. Natl. Acad. Sci.106, 7962-7967.

Gilbert, S. C. (2001). Virus-like particles as vaccine adjuvants. Molecular biotechnology, 19(2): 169-177.

Glauert, A. M., Dingle, J. T., and Lucy, J. A. (1962). Action of saponin on biological cell membranes. Nature, 196(4858), 953.

Gupta A. and Chaphalkar S. (2015). Vaccine adjuvants: the current necessity of life. Shiraz E- med J. doi: 10.17795/semj28061. 
Gupta, R. K., and Siber, G. R. (1995). Adjuvants for human vaccines - current status, problems and future prospects. Vaccine. 13(14), 1263-1276.

Halperin S.A., Dobson S., McNeil S., Langley J.M., Smit B., McCall-Sani R., Levitt D., Nest G.V., Gennevois D. and Eiden J.J. (2006). Comparison of the safety and immunogenicity of hepatitis B virus surface antigen co-administered with an immunostimulatory phosphorothioate oligonucleotide and a licensed hepatitis B vaccine in healthy young adults. Vaccine. 24, 20-26.

Heidelberg, J.F., Eisen, J.A., Nelson, W.C., Clayton, R.A., Gwinn, M.L., Dodson, R.J., Haft, D.H., Hickey, E.K., Peterson, J.D., Umayam, L. and Gill, S.R. (2000). DNA sequence of both chromosomes of the cholera pathogen Vibrio cholerae. Nature, 406(6795): 477.

Huleatt J.W., Jacobs A.R., Tang J., Desai P., Kopp E.B., Huang Y., Song L., Nakaar V. and Powell T.J. (2007). Vaccination with recombinant fusion proteins incorporating Toll-like receptor ligands induces rapid cellular and humoral immunity. Vaccine 25, 763-775.

Kawai, T., and Akira, S. (2010). The role of pattern-recognition receptors in innate immunity: update on Toll-like receptors. Nature immunology, 11(5), 373.

Krishnan, L., Dicaire, C. J., Patel, G. B., and Sprott, G. D. (2000). Archaeosome vaccine adjuvants induce strong humoral, cell-mediated, and memory responses: comparison to conventional liposomes and alum. Infection and immunity, 68(1), 54-63.

Lee S. and Nguyen M.T. (2015). Recent advances of vaccine adjuvants for infectious diseases. Immune network. 15, 51-57.

Liang F. and Lore, K. (2016). Local innate immune responses in the vaccine adjuvant-injected muscle. Clinical and Translational Immunology. doi:10.1038/ cti.2016.19
Lombard, M., Pastoret, P. P., and Moulin, A. M. (2007). A brief history of vaccines and vaccination. Revue Scientifique et Technique-Office International des Epizooties, 26(1): 29-48

Mohan T., Verma P. and Rao D. (2013). Novel adjuvants and delivery vehicles for vaccines development: A road ahead. Indian J Med Res. 138, 779-79.

Morein B., Sundquist B., Ho"glund S., Dalsgaard K. and Osterhaus A. (1984) Iscom, a novel structure for antigenic presentation of membrane proteins from enveloped viruses. Nature 308, 457-460.

Munks, M.W., McKee, A.S., MacLeod, M.K., Powell, R.L., Degen, J.L., Reisdorph, N.A., Kappler, J.W. and Marrack, P. (2010). Aluminum adjuvants elicit fibrindependent extracellular traps in vivo. Blood, pp.blood-2010.

Pasquale, A. D., Preiss, S., Silva, F. T. D., and Garçon, N. (2015). Vaccine adjuvants: from 1920 to 2015 and beyond. Vaccines. $3(2), 320-343$.

Podda A. (2001). The adjuvanted influenza vaccines with novel adjuvants: Experience with the MF59-adjuvanted vaccine. Vaccine.19, 2673-2680.

Ramon G. (1926). Procedes pour accroitre la production des antitoxins. Ann. Inst. Pasteur. 40, 1-10.

Reed S. Orr M. and Fox C. (2013). Key roles of adjuvants in modern vaccines. Nature Medicine. 19, 1597-1608.

Reed S., Bertholet S. Coler, R. and Friede M. (2008). New horizons in adjuvants for vaccine development. Trends in Immunology, 30, 23-32.

Roberts, M., Bacon, A., Rappuoli, R., Pizza, M., Cropley, I., Douce, G., Dougan, G., Marinaro, M., McGhee, J. and Chatfield, S.T.E.V.E.N. (1995). A mutant pertussis toxin molecule that lacks ADPribosyltransferase activity, PT-9K/129G, is an effective mucosal adjuvant for intranasally delivered proteins. Infection and immunity, 63(6): 2100-2108.

Satoshi Kashiwagi, Timothy Brauns, Jeffrey Gelfand and Mark C Poznansky. (2014). 
Laser vaccine adjuvants, Human Vaccines and Immunotherapeutics, 10(7): 1892-1907

Schijns V. and Brewer J. M. (2008). New views on immunopotentiators in modern vaccines. Expert Rev. Vaccines. 7,877879.

Schmidt S.T., Foged C., Korsholm K.S., Rades $\mathrm{T}$ and Dennis Christensen D. (2016). Liposome-based adjuvants for subunit vaccines: Formulation strategies for subunit antigens and immunostimulators. Pharmaceutics.8, 1-22.

Schwarz T.F., Horacek T., Knuf M., Damman H.G., Roman F., Drame M., Gillard P. and Jilg W. (2009). Single dose vaccination with AS03-adjuvanted H5N1 vaccines in a randomized trial induces strong and broad immune responsiveness to booster vaccination in adults. Vaccine, 27, 6284-6290.

Sérgio Jorge, Odir Antônio Dellagostin. (2017).

The development of veterinary vaccines: a review of traditional methods and modern biotechnology approaches. Biotechnology Research and Innovation. $1,6-13$

Singh, M., Chakrapani, A., and O'Hagan, D. (2007). Nanoparticles and microparticles as vaccine-delivery systems. Expert review of vaccines, 6(5), 797-808.
Sivakumar S. M., Safhi M. M., Kannadasan M. and Sukumaran N. (2011). Vaccine adjuvants - Current status and prospects on controlled release adjuvancity. Saudi Pharmaceutical Journal.19, 197-206.

Sivakumar, S. M., Safhi, M. M., Kannadasan, M., and Sukumaran, N. (2011). Vaccine adjuvants-current status and prospects on controlled release adjuvancity. Saudi Pharmaceutical Journal, 19(4), 197-206.

Soppimath, K. S., Aminabhavi, T. M., Kulkarni, A. R., and Rudzinski, W. E. (2001) Biodegradable polymeric nanoparticles as drug delivery devices. Journal of controlled release, 70(1-2), 1-20.

Spickler A. and Roth A. (2013). Adjuvants in Veterinary Vaccines: Modes of Action and Adverse Effects. J Vet Intern Med.17, 273-281.

Tandrup Schmidt, S., Foged, C., Smith Korsholm, K., Rades, T., and Christensen, D. (2016). Liposome-based adjuvants for subunit vaccines: formulation strategies for subunit antigens and immunostimulators. Pharmaceutics, 8(1), 7.

Tarr, P. I., Gordon, C. A., and Chandler, W. L. (2005). Shiga-toxin-producing Escherichia coli and haemolytic uraemic syndrome. The Lancet, 365(9464): 10731086.

\section{How to cite this article:}

Pushpa Makwana, Irshadullahkhan Kalyani, Dhruv Desai, Dharmesh Patel, Pramod Sakhare and Dushyant Muglikar. 2018. Role of Adjuvants in Vaccine Preparation: A Review. Int.J.Curr.Microbiol.App.Sci. 7(11): 972-988. doi: https://doi.org/10.20546/ijcmas.2018.711.113 